\title{
The epidemiology of cryptosporidiosis in Ontario, Canada following the introduction of PCR testing in 2018
}

\author{
Karen Johnson ${ }^{1}$, Palvasha Thayani ${ }^{2}$, Bryna Warshawsky ${ }^{1,3}$, Yvonne Whitfield $^{1}$, Jennifer Pritchard ${ }^{1}$, \\ Michelle Murti ${ }^{1,4 *}$
}

\section{Abstract}

Background: Cryptosporidiosis is reportable in Ontario, Canada. Detection by polymerase chain reaction (PCR) was introduced by a large community-based laboratory in August 2017, and in 2018, the incidence of reported cryptosporidiosis doubled compared to 2012-2016.

Methods: We assessed cases reported in 2018 for epidemiologic changes since the introduction of PCR testing.

Results: No outbreaks were identified in 2018, and $48 \%$ of cases were detected by PCR, suggesting that the observed increase was likely the result of PCR's higher sensitivity compared with previous detection method. From the pre to post-PCR periods, the proportion of female cases increased significantly, due mainly to cases diagnosed by PCR. A significant increase in mean age was also observed among cases diagnosed by microscopy and/or PCR in the postPCR period.

Conclusion: Our findings highlight the importance of assessing diagnostic methods when evaluating changes in reported rates. The observed changes in incidence will require ongoing monitoring and may require shorter baseline periods for aberration detection.

Suggested citation: Johnson KO, Thayani P, Warshawsky B, Whitfield Y, Pritchard J, Murti M. The epidemiology of cryptosporidiosis in Ontario, Canada following the introduction of PCR testing in 2018. Can Commun Dis Rep 2020;46(7/8):227-30. https://doi.org/10.14745/ccdr.v46i78a02

Keywords: Cryptosporidium, cryptosporidiosis, surveillance, PCR, polymerase chain reaction, epidemiology, outbreak detection

\section{Introduction}

Cryptosporidiosis is a diarrheal disease transmitted through fecal-oral contact with infected persons or animals. Although the mode of transmission varies, infection is most acquired through ingestion of drinking or recreational water that has been contaminated with Cryptosporidium oocysts (1). Cryptosporidiosis is traditionally diagnosed by microscopic examination of stool specimens and/or enzyme immunoassays for detection of antigens to Cryptosporidium spp. However, molecular methods including polymerase chain reaction (PCR) are increasingly used $(2,3)$. In Ontario, Canada, hospital laboratories, the provincial public health laboratory and privately operated community laboratories conduct diagnostic testing for cryptosporidiosis. Privately operated community laboratories diagnose the majority of cases reported. All laboratory-confirmed cases of cryptosporidiosis are reportable in Ontario (4), and reported cases are followed up by local boards of health that collect and report case information centrally to provincial public health officials through the integrated Public Health Information System (iPHIS).

Microscopy has been the main method of detection for cryptosporidiosis in Ontario. In August 2017, a large communitybased laboratory with collection centres across the province adopted multiplex PCR testing (5). Recent studies have shown the sensitivity of microscopy to range from $52 \%$ to $56 \%$ compared to PCR, which has a sensitivity of $100 \%(6,7)$.

In this article, we describe our investigation of an increase in reported cryptosporidiosis cases in 2018 with the aim of assessing the magnitude of the increase, determining the role 
of PCR testing in the increase and identifying if any shifts had occurred in the epidemiology of cryptosporidiosis since the implementation of PCR testing.

\section{Methods}

We conducted this assessment by comparing demographic, risk factor and outcome data from iPHIS for 2018 (the first full year following implementation of PCR testing) with the pre-PCR period of 2012-2016.

We extracted information on age, sex, outcome (hospitalization or death), reported symptoms, reporting laboratory (i.e. community based, hospital or public health) and method of diagnosis [i.e. PCR, microscopy, enzyme immunoassay (EIA) and culture-bacterial] from iPHIS for cryptosporidiosis cases with episode dates from January 1, 2012 to December 31, 2016 (pre-PCR) and from January 1 to December 31, 2018 (post-PCR).

Cases with no diagnostic data, cases diagnosed by PCR in the pre-PCR period and cases that had "culture-bacterial" (which reflect data entry errors) as the method of detection were excluded from all analyses except for overall case counts and rates. Social or behavioural risk factors, including history of travel outside the province, foodborne and waterborne exposures, and animal and person-to-person contact, for cases with episode dates in 2018, were also analyzed to determine whether there were any previously unrecognized outbreaks. All analyses were performed using the statistical package SAS Enterprise Guide version 7.1 ( $p<0.05$ considered statistically significant).

\section{Results}

Reported cases of cryptosporidiosis increased annually from 299 in 2012 to 429 in 2016, decreased to 391 in 2017 and then increased to 751 in 2018 (Figure 1). The annual rate of cryptosporidiosis in Ontario was 2.6 per 100,000 from 2012 to 2016 and 5.2 per 100,000 in 2018 ( $p<0.001)$. Based on 1,743 cases in the more detailed analysis, detection by PCR accounted for $48 \%$ of cryptosporidiosis cases reported in 2018 , while detection by microscopy decreased from $99.8 \%$ in $2012-2016$ to $52 \%$ in 2018 ( $p<0.001)$; EIA was the method of detection for three cases reported during the pre-PCR period $(0.2 \%)$.

Statistically significant overall and sex-specific increases in mean ages were also observed; the increases in the post-PCR period occurred among cases diagnosed by microscopy and by PCR (Table 1). Overall, the proportion of cases among females increased from $48.5 \%$ in the pre-PCR period to $55.4 \%$ in the post-PCR period $(p<0.009)$. Stratification of the post-PCR period showed that this shift in proportion from the pre-PCR period was driven by cases diagnosed by PCR $(60.8 \%$ in the post-PCR period, $p<0.001)$ as the proportion of female cases diagnosed
Figure 1: Number and incidence rate for all confirmed cases of cryptosporidiosis reported, Ontario, Canada, 2012-2018 $(n=2,928)^{a}$

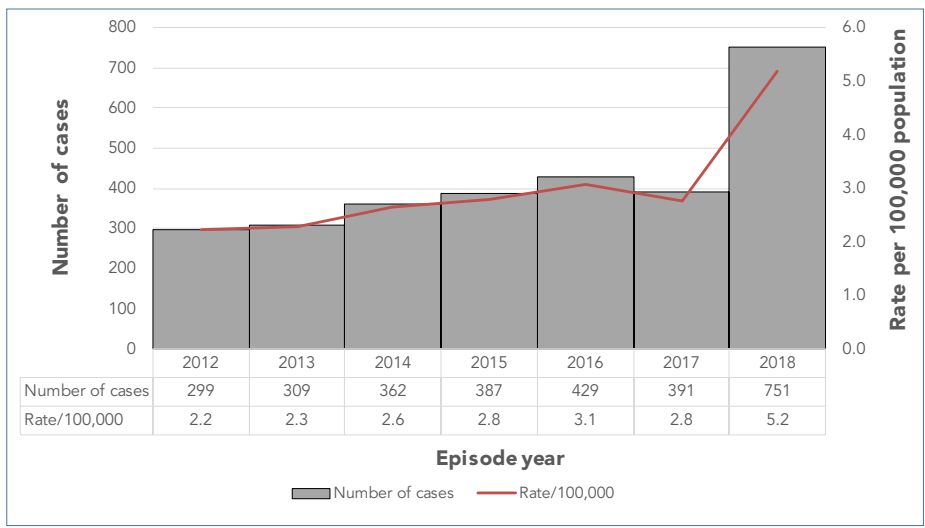

Population Estimates 1986-2017 and Population Projections 2017-2041, Ontario Ministry of Health, IntelliHEALTH ONTARIO, extracted: November 26, 2019

by microscopy in the post-PCR period $(50.4 \%)$ did not vary significantly from the pre-PCR period $(48.5 \%, p<0.580)$ (Table 1$)$.

From the pre to the post-PCR period, the proportion of hospitalized cases decreased from $5.5 \%$ to $2.1 \%(p<0.002)$. No deaths were recorded in either time period. Method of testing was not related to symptom status, although the proportion of asymptomatic cases was slightly higher among those diagnosed by PCR $(2.2 \%)$ and microscopy $(1.3 \%)$ in the post-PCR period relative to the pre-PCR period $(0.8 \%)$.

Based on the assessed risk factors, no commonalities were reported at the provincial level that suggested the occurrence of any outbreaks among non-travel cases in 2018 , or within the health units that had identified localized increases above their usual summer peak in incidence.

\section{Conclusion}

Our investigation identified a two-fold increase in the incidence of cryptosporidiosis cases in 2018, the first full year after the introduction of PCR testing in August 2017, compared to the pre-PCR period of 2012-2016. However, it remains unclear why the number of cases reported in 2017 decreased despite the introduction of PCR in that year and a gradually increasing trend in the previous five years. As only positive laboratory results are reportable in Ontario, we were not able to assess if the increase in 2018 was due to an increase in the number of cases, an increase in the number of tests ordered or an increase in positivity owing to PCR's higher sensitivity compared with previous detection method. Our investigation of the increase in 2018 also did not identify any common social and behavioural risk factors among domestic cases that were indicative of an outbreak. During the period of increase, clinical indications for testing and surveillance criteria for defining and reporting cases did not change, nor did healthcare providers have the option to 
Table 1: Cryptosporidiosis cases reported during the pre-PCR period (2012-2016) compared to cases reported in 2018, overall and stratified by diagnostic method $(n=1,743)^{\text {a }}$

\begin{tabular}{|c|c|c|c|c|c|c|c|c|c|c|c|}
\hline \multirow{3}{*}{$\begin{array}{c}\text { Case } \\
\text { characteristics }\end{array}$} & \multicolumn{2}{|c|}{ Pre-PCR period } & \multicolumn{9}{|c|}{$\begin{array}{c}\text { Post-PCR period } \\
(2018)^{\mathrm{b}}\end{array}$} \\
\hline & \multirow{2}{*}{\multicolumn{2}{|c|}{$\begin{array}{c}\text { Microscopy } \\
(n=1,209) \text { and } \\
\text { EIA }(n=3)\end{array}$}} & \multicolumn{3}{|c|}{ PCR } & \multicolumn{3}{|c|}{ Microscopy } & \multicolumn{3}{|c|}{ Microscopy and PCR } \\
\hline & & & \multicolumn{2}{|c|}{$n=255$} & $p$ value ${ }^{c}$ & \multicolumn{2}{|c|}{$n=276$} & $p$ value ${ }^{c}$ & \multicolumn{2}{|c|}{$n=531$} & $p$ value \\
\hline Diagnosed (\%) & \multicolumn{2}{|c|}{$\begin{array}{c}\text { Microscopy }(99.8 \%) \\
\text { EIA }(0.2 \%)\end{array}$} & \multicolumn{3}{|c|}{$48.0 \%$} & \multicolumn{2}{|r|}{$52 \%$} & - & \multirow{2}{*}{\multicolumn{2}{|c|}{$100 \%$}} & - \\
\hline \multicolumn{12}{|l|}{ Mean age (years) } \\
\hline Female & \multicolumn{2}{|r|}{23.3} & \multicolumn{2}{|r|}{27.3} & $<0.005$ & \multicolumn{2}{|r|}{28.4} & $<0.001$ & & & $<0.001$ \\
\hline Male & \multicolumn{2}{|r|}{20.9} & \multicolumn{2}{|r|}{26.4} & $<0.002$ & \multicolumn{2}{|r|}{24.9} & $<0.011$ & \multicolumn{2}{|r|}{25.5} & $<0.001$ \\
\hline Total $^{d}$ & \multicolumn{2}{|r|}{22.1} & \multicolumn{2}{|r|}{26.9} & $<0.001$ & \multicolumn{2}{|r|}{26.6} & $<0.001$ & \multicolumn{2}{|r|}{26.8} & $<0.001$ \\
\hline \multicolumn{12}{|l|}{ Sex, n (\%) ${ }^{d}$} \\
\hline Female & $(48.5)$ & $(48.5)$ & 155 & (60.8) & $<0.001$ & 139 & $(50.4)$ & $<0.580$ & 294 & $(55.4)$ & $<0.009$ \\
\hline Male & 620 & $(51.2)$ & 100 & $(39.2)$ & $<0.001$ & 136 & (49.3) & $<0.573$ & 236 & $(44.4)$ & $<0.010$ \\
\hline Hospitalized, n (\%) & 67 & $(5.5)$ & 4 & $(1.6)$ & $<0.008$ & 7 & $(2.5)$ & $<0.040$ & 11 & $(2.1)$ & $<0.001$ \\
\hline Symptom status ${ }^{e}$ & & & & & & & & & & & \\
\hline Asymptomatic, n (\%) & 9 & $(0.8)$ & 5 & $(2.2)$ & $<0.084$ & 3 & (1.3) & $<0.461$ & 8 & (1.7) & $<0.129$ \\
\hline
\end{tabular}

Abbreviations: EIA, enzyme immunoassay; PCR, polymerase chain reaction, -, no data

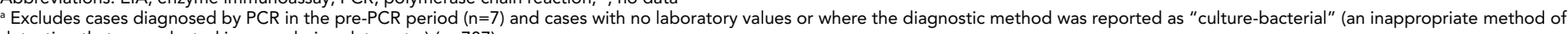
detection that was selected in error during data entry) ( $n=787)$

${ }^{b}$ No cases were diagnosed by EIA in 2018

c $P$ values based on appropriate tests for proportions (Pearson chi-square and Fisher exact test) and means ( $t$-test). The $p$ values indicate the probability of finding the observed values for the comparisons between the combined methods of detection in the pre-PCR period and the post-PCR period (PCR, microscopy and PCR and microscopy combined)

"Excludes cases where sex was reported as "other" or "unknown"

excludes cases with no data on symptoms and cases with symptoms that were classified as not clinically compatible with cryptosporidiosis (e.g. cough)

specify their preferred diagnostic method (e.g. microscopy, EIA or PCR) when ordering tests. Given these factors, it is possible that the increase in 2018 resulted, at least partially, from PCR's higher sensitivity, which resulted in the detection of cases that would have otherwise remained undiagnosed by microscopy or EIA.

With the increase in the reported rate of cryptosporidiosis, there was also an increase in the proportion of female cases in 2018, driven by PCR diagnoses. One possible hypothesis is that the baseline test positivity rate for females was lower compared to males and subsequently more affected by the higher sensitivity of PCR testing. In contrast, the increase in mean age among cases diagnosed by both PCR and microscopy in 2018 may reflect an underlying shift in the age distribution of persons tested in 2018 compared to 2012-2016. We would require data on the age and sex of persons that submitted specimens for testing, testing volumes and positivity rates to fully assess these changes in the demographics of cryptosporidiosis cases.

The shift towards fewer hospitalized and more asymptomatic cases after the introduction of PCR testing reflects PCR's higher sensitivity and use in community laboratories that test nonhospitalized patients. Similar trends among microscopy-detected cases were also observed, suggesting that factors other than the introduction of PCR testing may have influenced the shifts in hospital and symptom status in 2018. However, given the small proportions of cases overall that were hospitalized or asymptomatic, caution must be exercised in drawing conclusions from these limited post-PCR data.

Our investigation demonstrated that the traditional approach to examining incidence over time for trends and outbreak detection must be coupled with a review of other data elements such as diagnostic methods and risk factor information, which can indicate whether increases are artifacts, due to increases in sporadic cases or attributable to a specific source. We anticipate that the adoption of PCR testing for diagnosing cryptosporidiosis will occur at different times by the various diagnostic laboratories in Ontario, resulting in staggered increases in incidence. These changes will require continuous reassessment of baseline values for accurate interpretation of trends for aberration detection and timely outbreak detection. With time, we expect the emergence of a more stable and long-standing baseline at a higher incidence rate due to the more widespread use of PCR. As our investigation reflects one year of PCR testing, analysis of additional years of data is required to understand the clinical and public health relevance of cases detected by this method.

\section{Authors' statement}

KJ - Data analysis, Writing - review \& editing PT — Data analysis, Writing - review \& editing BW - Data review, Writing - review \& editing 
YW - Data review, Writing - review \& editing

JP — Project administration, Writing - review \& editing

MM - Writing - original draft, Writing - review \& editing

\section{Competing interests}

None.

\section{Funding}

This work was supported by Public Health Ontario.

\section{Acknowledgements}

We thank L Friedman from Public Health Ontario for providing copy editing and reference management.

\section{References}

1. Heymann DL, editor. Control of communicable diseases manual. 19th ed. Washington (DC): American Public Health Association; 2008.

2. van Lieshout $L$, Roestenberg M. Clinical consequences of new diagnostic tools for intestinal parasites. Clin Microbiol Infect 2015;21(6):520-8. DOI PubMed

3. Adeyemo FE, Singh G, Reddy P, Stenström TA. Methods for the detection of Cryptosporidium and Giardia: from microscopy to nucleic acid based tools in clinical and environmental regimes. Acta Trop 2018;184:15-28.

DOI PubMed
4. Ministry of Health and Long-term Care. Infectious diseases protocol: Appendix B: Provincial case definitions for diseases of public health significance; Disease: Cryptosporidiosis. Toronto (ON): Government of Ontario; 2019. http://www. health.gov.on.ca/en/pro/programs/publichealth/oph_ standards/docs/crypto_cd.pdf

5. LifeLabs. Client notice update - LifeLabs introduces PCR testing for detection of parasites in stool samples. Toronto (ON): LifeLabs; 2017 Aug 24 (accessed 2018-07-26). https:// www.lifelabs.com/client-notice-update-lifelabs-introduces -pcr-testing-for-detection-of-parasites-in-stool-samples/

6. Stark D, Al-Qassab SE, Barratt JL, Stanley K, Roberts T, Marriott D, Harkness J, Ellis JT. Evaluation of multiplex tandem real-time PCR for detection of Cryptosporidium spp., Dientamoeba fragilis, Entamoeba histolytica, and Giardia intestinalis in clinical stool samples. J Clin Microbiol 2011;49(1):257-62. DOI PubMed

7. Laude A, Valot S, Desoubeaux G, Argy N, Nourrisson C, Pomares C, Machouart M, Le Govic Y, Dalle F, Botterel F, Bourgeois N, Cateau E, Leterrier M, Le Pape P, Morio F. Is real-time PCR-based diagnosis similar in performance to routine parasitological examination for the identification of Giardia intestinalis, Cryptosporidium parvum/ Cryptosporidium hominis and Entamoeba histolytica from stool samples? Evaluation of a new commercial multiplex PCR assay and literature review. Clin Microbiol Infect 2016;22(2):190.e1-8. DOI PubMed 